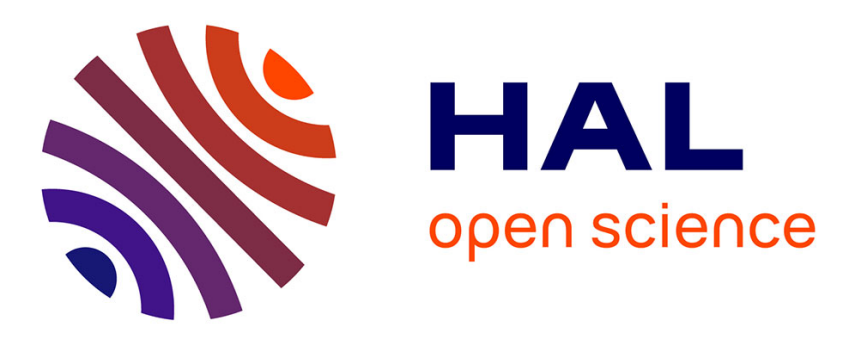

\title{
Numerical simulation of polymer flows using non-conforming finite elements
}

Didier Graebling, Julie Joie

\section{To cite this version:}

Didier Graebling, Julie Joie. Numerical simulation of polymer flows using non-conforming finite elements. Computers and Fluids, 2013, 79, pp.178 - 189. 10.1016/j.compfluid.2013.03.012 . hal03482592

\section{HAL Id: hal-03482592 \\ https://hal.science/hal-03482592}

Submitted on 5 Jan 2022

HAL is a multi-disciplinary open access archive for the deposit and dissemination of scientific research documents, whether they are published or not. The documents may come from teaching and research institutions in France or abroad, or from public or private research centers.
L'archive ouverte pluridisciplinaire HAL, est destinée au dépôt et à la diffusion de documents scientifiques de niveau recherche, publiés ou non, émanant des établissements d'enseignement et de recherche français ou étrangers, des laboratoires publics ou privés. 


\title{
Numerical simulation of polymer flows using non-conforming finite elements
}

\author{
Julie Joie ${ }^{\mathrm{a}, \mathrm{b}}$, Didier Graebling ${ }^{\mathrm{d}, \mathrm{c}, *}$ \\ a Institut de Mathématiques de Bordeaux, UMR CNRS 5251 Université Bordeaux 1, 351 cours de la Libération, 33405 Talence Cedex (France) \\ ${ }^{b}$ INRIA Bordeaux-Sud-Ouest - Équipe Projet MC2 (France) \\ ${ }^{c}$ INRIA Bordeaux-Sud-Ouest - Équipe Projet Concha (France) \\ ${ }^{d}$ IPREM/EPCP, UMR CNRS 5154 Université de Pau et des Pays de l'Adour 2 avenue Angot 64053 Pau Cedex 9 (France)
}

\begin{abstract}
In this paper, we are interested in the simulation of polymer flows for high-Weissenberg numbers. The highWeissenberg number problem (HWNP) is one of the main difficulties encountered for the numerical simulation of such flows. We develop a numerical approach for two non-linear models: the affine Phan-Thien and Tanner model and the Giesekus model. We consider the 2D case and triangular and quadrilateral meshes. The velocity and the pressure are approximated by non-conforming finite elements while the stress tensor is approximated by $P_{0}$ totally discontinuous finite elements. We have considered three popular test-cases: a simple channel, a 4:1 abrupt contraction and a cylinder. Comparisons with analytical solutions and experiences are performed, illustrating the good behaviour of our code. Moreover, for the Oldroyd-B model, we have performed comparisons of drag values with data given in the literature. We have been able to obtain simulations for large values of Weissenberg number ( $\mathfrak{B i}>21$ for the 4:1 contraction), our approach gives a realistic description of polymer flows.
\end{abstract}

Keywords: Phan-Thien and Tanner model, Giesekus model, Weissenberg number, nonconforming finite element method

\section{Introduction}

Despite numerous efforts, computational nonNewtonian fluid mechanics is still a very challenging research area. The high-Weissenberg number problem (HWNP) is one of the main difficulties encountered for the numerical simulation of polymer flows. The source of the problem is the breakdown in convergence of the algorithms at critical values of the Weissenberg number. The frustratingly low value of the Weissenberg number limits the CFD use for the polymer processing industry [19, 36].

Besides this major issue, there are two other aspects that have to be carefully treated by the finite element discretization: the choice of approximation spaces satisfying the Babuška-Brezzi compatibility condition and the treatment of the convective terms.

Several well-posed mixed finite element approximation have been developed during the last decades. Most

\footnotetext{
${ }^{*}$ Corresponding author

Email addresses: julie.joie@inria.fr(Julie Joie), didier.graebling@univ-pau.fr (Didier Graebling)

Preprint submitted to Computers $\mathcal{E}$ Fluids
}

approaches consist in adding ellipticity on the momentum equation in order to stabilize the scheme. King et al. [21] introduce the Elasticity Elliptic Momentum Equation (EEME) method, which is a reformulation of the momentum equation that makes the elliptic character of this equation explicit. Another popular method is the Elastic-Viscous Split Stress (EVSS) finite element method introduced by Rajagopalan et al. [33], which consists in splitting the stress tensor into a viscous part and an elastic part and to perform a change of variables. The Adaptative Viscous Split Stress (AVSS) method of Sun, Phan-Thien and Tanner [35], introduced another way to perform this change of variable. Nevertheless, this change of variable is not possible with all the constitutive equation. To overcome this problem, Guénette and Fortin [16] introduced the Discrete Elastic-Viscous Split Stress (DEVSS) finite element method, where the same split is perfomed, but no change of variable is needed.

Concerning the discretization of the convective term, there exist two main approaches: one based on the Streamline-Upwind method (SU or SUPG) and the other on discontinuous Galerkin methods $(\mathrm{dG})$ follow-

September 2, 2015 
ing the Lesaint-Raviart scheme. The first class of methods consists in adding streamline upwind artificial diffusivity and was first applied to the computation of viscoelastic flows in 1987 by Marchal and Crochet [26]. The dG method is based on the LesaintRaviart method [24] and was first applied to a viscoelastic liquid by Fortin and Fortin [13]. An advantage of this method, is that the velocity-stress tensor spaces compatibility condition required for the three field Stokes problem, can be easely satisfied. Morover, the dG methods are known to be easy to implement.

For a complete review of these methods one can refers to $[1,2,28]$.

Recently, it has been shown that the breakdown in convergence of the algorithms is related with the lack of positivity of the so-called conformation tensor at the discrete level [37, 23]. The conformation tensor can be interpreted as a tensorial measure of the molecular orientation and stretching of the chain. This tensor denotes the average of the dyadic product of the end-toend vector of a polymer chain. Grmela introduced a class of rheological models based on the conformation tensor [17]. In these models, the conformation tensor is assumed to be symmetric and positive definite. In the last few years, numerical schemes preserving the positive definiteness of the discrete conformation tensor have been proposed in the literature based on the approach of Fattal and Kupferman [12]. They consider a log-conformation formulation of the constitutive equation written in terms of $\boldsymbol{\psi}=\ln \boldsymbol{C}$ and then put $\boldsymbol{C}_{h}=\mathrm{e}^{\psi_{h}}$. This method has been widely used $[18,9,22]$. An alternate log-conformation formulation has been introduced by Coronado et al. [6]. Those methods lead to stongly nonlinear reformulations of the considered problems and therefore, their computation is very costly. Lee and Xu employed the framework of Riccati equations to preserve the discrete positivity [23].

Among the rheological models developed for describe the polymer liquid flows, the Giesekus model is one of the most realistic [14, 15]. This model presents two main advantages. First, it yields a realistic behaviour for all flows except for the biaxial exten$\operatorname{sion}^{1}[20]$. Second, only two material parameters, the relaxation time $\lambda$ and the viscosity $\eta$, are needed to describe the model. These parameters can be easily determined using dynamic rheology experiments. However, the Giesekus constitutive law is strongly nonlinear since it involves a quadratic term in the stress tensor. Here,

\footnotetext{
${ }^{1}$ For this flow, the expected behaviour is possible but is not in good agreement with the experimental data.
}

we also consider the simplified or affine Phan-ThienTanner model [30, 31].

In this paper, we consider a low order nonconforming finite element method to approach the velocity and the pressure and $\mathrm{dG}$ finite elements to approach the stress tensor. The presented methodology is implemented in the academic $\mathrm{C}++$ library Concha ${ }^{2}$. To validate the code, convergence tests and comparisons with analytical solutions are performed. We have also computed the cylinder drag values for an Oldroyd-B liquid in order to compare our numerical scheme with other proposed in the literature. For the $4: 1$ abrupt contraction geometry, we present velocities and stress comparisons between experimental data [32] and our code. Finally, this geometry allows to show simulations for high Weissenberg numbers.

The paper is structured as follows: in section 2, we present the rheological models used. The section 3 is devoted to the description of the numerical schemes. In the last section, we present the numerical results.

\section{Governing equations}

In the case of incompressible isothermal flows, the motion of a liquid is described by:

- the mass conservation law,

$$
\nabla \cdot \boldsymbol{u}=0
$$

where $\boldsymbol{u}$ is the velocity of the liquid.

- the momentum conservation law,

$$
\rho\left(\frac{\partial}{\partial t} \boldsymbol{u}+\boldsymbol{u} \cdot \nabla \boldsymbol{u}\right)-\nabla \cdot \boldsymbol{\tau}+\nabla p=\mathbf{0}
$$

where $\tau, p$ and $\rho$ are respectively the extra-stress tensor, the pressure and the density of the fluid.

- and a constitutive equation.

The rheological behaviour of a polymer liquid can be described by two types of differential constitutive equations:

- the quasi-linear differential models:

$$
\boldsymbol{\tau}+\lambda \stackrel{\square}{\boldsymbol{\tau}}_{a}=2 \eta \boldsymbol{D}
$$

with $\bar{\tau}_{a}$ the Gordon-Schowalter convected derivative of the extra-stress tensor.

\footnotetext{
${ }^{2}$ http://sites.google.com/site/conchapau/
} 
- the nonlinear differential models:

$$
f(\boldsymbol{\tau})+\lambda \stackrel{\nabla}{\boldsymbol{\tau}}=2 \eta \boldsymbol{D}
$$

with $f(\tau)$ a nonlinear function of the extra-stress tensor.

$\boldsymbol{D}$ is the Oldroyd strain-rate tensor given by:

$$
\boldsymbol{D}=\frac{1}{2}\left\{\boldsymbol{\nabla u}+(\boldsymbol{\nabla u})^{t}\right\} .
$$

$\eta$ and $\lambda$ are respectively the zero-shear viscosity and the relaxation time of the polymer liquid.

The Gordon-Schowalter convected derivative of the tensor $\boldsymbol{A}$ is defined by the following relationship:

$$
\begin{aligned}
\stackrel{\boldsymbol{A}}{a}== & \frac{\partial}{\partial t} \boldsymbol{A}+\boldsymbol{u} \cdot \boldsymbol{\nabla} \boldsymbol{A} \\
& +\boldsymbol{A} \cdot \boldsymbol{\Omega}-\boldsymbol{\Omega} \cdot \boldsymbol{A}-a\{\boldsymbol{A} \cdot \boldsymbol{D}+\boldsymbol{D} \cdot \boldsymbol{A}\}
\end{aligned}
$$

where $a$ is a parameter $\in[-1,1] . \Omega$ is the vorticiy tensor defined by:

$$
\mathbf{\Omega}=\frac{1}{2}\left\{(\boldsymbol{\nabla} \boldsymbol{u})^{t}-\boldsymbol{\nabla} \boldsymbol{u}\right\}
$$

According to the chosen values for $a$, we obtain:

$$
\begin{aligned}
& \text { ( the upper-convected derivative for } a=1 \text { : } \\
& \stackrel{\nabla}{\boldsymbol{\tau}}=\frac{\partial}{\partial t} \boldsymbol{\tau}+\boldsymbol{u} \cdot \boldsymbol{\nabla} \boldsymbol{\tau}-\left\{\boldsymbol{\tau} \cdot \boldsymbol{\nabla} \boldsymbol{u}+(\boldsymbol{\nabla u})^{t} \cdot \boldsymbol{\tau}\right\} \\
& \stackrel{\circ}{\boldsymbol{\tau}}=\frac{\partial}{\partial t} \boldsymbol{\tau}+\boldsymbol{u} \cdot \boldsymbol{\nabla} \boldsymbol{\tau}+\boldsymbol{\tau} \cdot \boldsymbol{\Omega}+\boldsymbol{\Omega} \cdot \boldsymbol{\tau} \\
& \stackrel{\Delta}{\boldsymbol{\tau}}=\frac{\partial}{\partial t} \boldsymbol{\tau}+\boldsymbol{u} \cdot \boldsymbol{\nabla} \boldsymbol{\tau}+\boldsymbol{\tau} \cdot(\boldsymbol{\nabla} \boldsymbol{u})^{t}+\boldsymbol{\nabla} \boldsymbol{u} \cdot \boldsymbol{\tau}
\end{aligned}
$$

If we replace the time derivative by an objective time derivative, a linear model such as the Maxwell model could be transformed into a quasi-linear model. The Oldroyd-B model can be regarded as an extension of the Upper Convected Maxwell (UCM) model. The deviatoric term of the stress is split into a polymeric part and a solvent or Netwonian part: $\tau=\tau_{p}+\tau_{s}$. The constitutive equation of this model is given by:

$$
\begin{aligned}
\boldsymbol{\tau}_{s} & =2 \eta_{s} \boldsymbol{D} \\
\boldsymbol{\tau}_{p}+\lambda \boldsymbol{\tau}_{p} & =2 \eta_{p} \boldsymbol{D}
\end{aligned}
$$

The viscosity of this liquid is defined by: $\eta=\eta_{s}+\eta_{p}$.

In this work, we consider two non-linear viscoelastic liquids: the simplified version of the Phan-ThienTanner model [30, 31] and the Giesekus model [14, 15].

According to the choice of the function $f(\tau)$ in (4), we obtain:
- the simplified or affine Phan-Thien-Tanner model (PTT):

$$
\begin{aligned}
f(\tau) & =\left(1+\frac{\epsilon \lambda}{\eta} \operatorname{tr}\{\tau\}\right) \tau \\
& \Rightarrow \quad \tau+\frac{\epsilon \lambda}{\eta} \operatorname{tr}\{\tau\} \tau+\lambda \stackrel{\nabla}{\tau}=2 \eta D
\end{aligned}
$$

where $\epsilon$ is a non-dimensional adjustable parameter called the extensional parameter.

- the Giesekus model:

$$
\begin{aligned}
f(\boldsymbol{\tau}) & =\boldsymbol{\tau}+\frac{\alpha}{G} \boldsymbol{\tau} \cdot \boldsymbol{\tau} \\
& \Rightarrow \boldsymbol{\tau}+\frac{\alpha}{G} \boldsymbol{\tau} \cdot \boldsymbol{\tau}+\lambda \stackrel{\nabla}{\boldsymbol{\tau}}=2 \eta \boldsymbol{D}
\end{aligned}
$$

where $\alpha$ is a constant $\in[0,1]$ and $G=\eta / \lambda$ is called the elastic modulus.

For a steady shear flow, the simplified form of the PTT model predicts a pseudoplastic behaviour, a first normal-stress difference function of the shear-rate and a zero second normal-stress difference. In the case of an elongational flow, this model describes suitably the polymer melt behaviour. The parameter $\epsilon$ imposes an upper limit to the elongational viscosity which becomes inversely proportional to this parameter.

The Giesekus constitutive equation describes accurately a large panel of material functions. In the case $\alpha=0.5$, this relatively simple constitutive equation predicts a pseudoplastic behaviour with the first and second normal-stress differences function of the shear-rate. This model predicts, in the case of an elongational flow, a Troutonian behaviour and a strain hardening with a finite asymptotic value. Setting $\alpha=0$ reduces the model to the Upper Convected Maxwell model.

The polymer flow is characterized by the Weissenberg number $\mathfrak{M i}$ defined as follows:

$$
\mathfrak{W i}=\lambda \dot{\gamma}
$$

where $\dot{\gamma}$ is the shear strain. In our case, it is defined for a Newtonian fluid.

\section{Numerical method}

Let us first introduce some useful notations. We consider an open bounded domain $\Omega$ of $\mathbb{R}^{2}$ and a regular family of triangulation $\left(\mathcal{T}_{h}\right)_{h>0}$ consisting of triangles or quandrangles. We agree to denote by $\varepsilon_{h}^{\text {int }}$ the set of internal edges of $\mathcal{T}_{h}$, by $\varepsilon_{h}^{\partial}$ the set of edges situated on the 
boundary $\partial \Omega$ and we put $\varepsilon_{h}=\varepsilon_{h}^{i n t} \cup \varepsilon_{h}^{\partial}$. As usually, let $h_{T}$ be the diameter of the triangle $T$ and let $h=\max _{T \in \mathcal{T}_{h}} h_{T}$.

On every edge $e$ belonging to $\varepsilon_{h}^{i n t}$, such that $\{e\}=$ $\partial T^{i} \cap \partial T^{j}$, we define once and for all the unit normal n. For a given function $\varphi$ with $\varphi_{\mid T_{i}} \in C\left(T_{i}\right)(1 \leq i \leq 2)$, we define on $e: \varphi^{e x t}(\boldsymbol{x})=\lim _{\varepsilon \rightarrow 0} \varphi(\boldsymbol{x}-\varepsilon \boldsymbol{n}), \varphi^{i n}(\boldsymbol{x})=$ $\lim _{\varepsilon \rightarrow 0} \varphi(\boldsymbol{x}+\boldsymbol{\varepsilon n})$ as well as the jump $[\varphi]=\varphi^{\text {ext }}-\varphi^{i n}$. If $e \in \varepsilon_{h}^{\partial}, \boldsymbol{n}$ is the outward unit normal and $[\varphi]$ is the trace of $\varphi$ on $e$. We agree to denote the $L^{2}(e)$ orthogonal projection of a given function $\varphi \in L^{2}(e)$ on $P_{k}(k \in \mathbb{N})$ by $\pi_{k} \varphi$ where $P_{k}$ is the polynomial space of maximum degree $k$. As usually, we denote by $\varphi^{-}=\min \{0, \varphi\}$ the negative part of $\varphi$ and we set $\varphi^{+}=\varphi-\varphi^{-}$. We denote by $c$ any constant independent of $h, \eta$ and the stabilization parameters. We shall use the notation $\boldsymbol{\tau}: \boldsymbol{\theta}=\sum_{i, j=1}^{2} \tau_{i j} \theta_{i j}$.

We consider a velocity-pressure-stress tensor formulation of the previous models. Our choice of the discrete spaces is based on a previous analysis on Newtonian flows [3]. Indeed, we have studied a dG approximation of the underlying three-fields Stokes problem related with a non-conforming method. We have obtained theoretical and numerical results proving the stability of this method. However, the computation is very costly and therefore, the method is not well-adapted for threefields formulations. Based on to the optimal theoretical and numerical results obtained, we have chosen to use here a combination of these two finite element methods.

We only detail the numerical schemes for the Giesekus model with $\alpha=0.5$ composed by the equations (1), (2) and (10). Similar formulations can be obtained for the other considered models. We complete the model by adding boundary conditions: $\boldsymbol{u}=\boldsymbol{g}$ on $\partial \Omega$ and $\boldsymbol{\tau}=\tau^{D}$ on the inflow boundary $\partial \Omega^{-}\{x \in \partial \Omega ; \boldsymbol{u}(x)$. $\boldsymbol{n}(x)<0\}$. We take $\boldsymbol{f} \in\left(L^{2}(\Omega)\right)^{2}, \boldsymbol{g} \in\left(H^{1 / 2}(\partial \Omega)\right)^{2}$ and $\boldsymbol{\tau}^{D} \in \boldsymbol{L}_{\text {sym }}^{2}\left(\partial \Omega^{-}\right)$, with:

$$
\boldsymbol{L}_{s y m}^{2}(\omega)=\left\{\boldsymbol{\tau}=\left(\tau_{i j}\right)_{1 \leq i, j \leq 2} ; \boldsymbol{\tau}=\boldsymbol{\tau}_{i j}=\boldsymbol{\tau}_{j i}, \tau_{i j} \in L^{2}(\omega)\right\} .
$$

We consider here the steady case and we present the corresponding discretization for the triangular case first, then for the quadrilateral case.

\subsection{Triangular case}

We first consider triangular elements. Let us now describe the approximation of each of the variable. The velocity and the pressure are approximated by non-conforming finite elements of Crouzeix-Raviart [7] while the stress tensor is approximated by $P_{0}$ discontinuous finite elements (Fig. 1). Thus, we introduce the corresponding discrete spaces:

$$
\begin{aligned}
\boldsymbol{V}_{h}=\left\{\boldsymbol{v} \in \boldsymbol{L}^{2}(\Omega) ;(\boldsymbol{v})_{/ T} \in \boldsymbol{P}_{1},\right. \\
\left.\qquad T \in \mathcal{T}_{h} \text { and }\left[\pi_{0} \boldsymbol{v}\right]_{/ e}=0, \forall e \in \varepsilon_{h}\right\}, \\
\boldsymbol{V}_{h}^{\boldsymbol{g}}=\left\{\boldsymbol{v}_{h} \in \boldsymbol{V}_{h} ; \int_{e} \boldsymbol{v}_{h} d s=\int_{e} \boldsymbol{g} d s \quad \forall e \in \varepsilon_{h}^{\partial}\right\} \\
Q_{h}=\left\{q \in L_{0}^{2}(\Omega) ;(q)_{/ T} \in P_{0}, \quad \forall T \in \mathcal{T}_{h}\right\}, \\
\boldsymbol{X}_{h}=\left\{\boldsymbol{\sigma} \in \boldsymbol{L}_{\text {sym }}^{2}(\Omega) ;(\boldsymbol{\sigma})_{/ T} \in \boldsymbol{P}_{0}, \quad \forall T \in \mathcal{T}_{h}\right\},
\end{aligned}
$$

with

$$
L_{0}^{2}(\Omega)=\left\{q \in L^{2}(\Omega): \int_{\Omega} q d x=0\right\} .
$$

Figure 1: Degrees of freedom for triangular case.

The finite element method requires a weak formulation of the system of PDE's to be solved. The discrete formulation can be written as follows:

$$
\left\{\begin{array}{cccc}
\left(\boldsymbol{u}_{h}, p_{h}, \boldsymbol{\tau}_{h}\right) \in \boldsymbol{V}_{h}^{g} \times Q_{h} \times \boldsymbol{X}_{h} & & & \\
a\left(\boldsymbol{u}_{h}, \boldsymbol{u}_{h} ; \boldsymbol{v}_{h}\right)+b\left(p_{h}, \boldsymbol{v}_{h}\right) & & & \\
\quad+c_{0}\left(\boldsymbol{v}_{h}, \boldsymbol{\tau}_{h}\right) & = & f\left(\boldsymbol{v}_{h}\right) & \forall \boldsymbol{v}_{h} \in \boldsymbol{V}_{h}^{\mathbf{0}} \\
b\left(q_{h}, \boldsymbol{u}_{h}\right) & = & 0 & \forall q_{h} \in Q_{h} \\
c\left(\boldsymbol{u}_{h}, \boldsymbol{\tau}_{h} ; \boldsymbol{\sigma}_{h}\right)+d\left(\boldsymbol{\tau}_{h}, \boldsymbol{\tau}_{h} ; \boldsymbol{\sigma}_{h}\right) & = & l\left(\boldsymbol{\sigma}_{h}\right) & \forall \boldsymbol{\sigma}_{h} \in \boldsymbol{X}_{h}
\end{array}\right.
$$

The form $a(\cdot, \cdot ; \cdot)$ can be decomposed into two parts:

$$
a\left(\boldsymbol{u}_{h}, \boldsymbol{u}_{h} ; \boldsymbol{v}_{h}\right)=a_{0}\left(\boldsymbol{u}_{h}, \boldsymbol{u}_{h} ; \boldsymbol{v}_{h}\right)+\gamma a_{1}\left(\boldsymbol{u}_{h}, \boldsymbol{v}_{h}\right) .
$$

The term $a_{0}(\cdot, \cdot ; \cdot)$ represents the approximation of the following convective term:

$$
a_{0}\left(\boldsymbol{u}_{h}, \boldsymbol{u}_{h} ; \boldsymbol{v}_{h}\right)=\sum_{T \in \mathcal{T}_{h}} \int_{T} \rho \boldsymbol{u}_{h} \nabla \boldsymbol{u}_{h} \cdot \boldsymbol{v}_{h} d x
$$

plus, eventually an upwinding stabilization whereas the linear form $a_{1}(\cdot, \cdot)$ is defined by:

$$
a_{1}\left(\boldsymbol{u}_{h}, \boldsymbol{v}_{h}\right)=\eta \sum_{e \in \varepsilon_{h}^{\text {int }}} \frac{1}{|e|} \int_{e}\left[\left(\boldsymbol{u}_{h} \cdot \boldsymbol{n}\right)\right]\left[\left(\boldsymbol{v}_{h} \cdot \boldsymbol{n}\right)\right] d s
$$


and $\gamma$ is a stabilization parameter. This term stabilizes the formulation thanks to a discrete Korn type inequality for discontinuous spaces [3].

The form $b(\cdot, \cdot)$ is the classical one involving the divergence of the velocity:

$$
b\left(q_{h}, \boldsymbol{v}_{h}\right)=-\sum_{T \in \mathcal{T}_{h}} \int_{T} q_{h} \boldsymbol{\nabla} \cdot \boldsymbol{v}_{h} d x
$$

The nonlinear forms $c(\cdot, \cdot ; \cdot)$ and $d(\cdot, \cdot ; \cdot)$ are defined by:

$$
\begin{aligned}
& c(\cdot, \cdot ; \cdot)=-2 \eta c_{0}(\cdot, \cdot)+c_{1}(\cdot, \cdot ; \cdot)+c_{2}(\cdot, \cdot ; \cdot), \\
& d(\cdot, \cdot ; \cdot)=d_{0}(\cdot, \cdot)+\alpha d_{1}(\cdot, \cdot ; \cdot) .
\end{aligned}
$$

Here above, $c_{0}(\cdot, \cdot)$ is the linear form:

$$
c_{0}\left(\boldsymbol{\tau}_{h}, \boldsymbol{v}_{h}\right)=\sum_{T \in \mathcal{T}_{h}} \int_{T} \boldsymbol{\tau}_{h}: \boldsymbol{D}\left(\boldsymbol{v}_{h}\right) d x,
$$

$c_{2}(\cdot, \cdot ;)$ represents the objective derivative:

$$
\begin{aligned}
c_{2}\left(\boldsymbol{u}_{h}, \boldsymbol{\tau}_{h} ; \boldsymbol{\sigma}_{h}\right)=-\lambda & \sum_{T \in \mathcal{T}_{h}} \int_{T} \boldsymbol{\tau}_{h} \boldsymbol{\nabla} \boldsymbol{u}_{h}: \boldsymbol{\sigma}_{h} d x \\
& -\lambda \sum_{T \in \mathcal{T}_{h}} \int_{T}\left(\boldsymbol{\nabla} \boldsymbol{u}_{h}\right)^{t} \boldsymbol{\tau}_{h}: \boldsymbol{\sigma}_{h} d x,
\end{aligned}
$$

and $c_{1}(\cdot, \cdot ; \cdot)$ is the approximation of the convective term $\boldsymbol{u} \cdot \nabla \boldsymbol{\tau}$. We follow the approach of Lesaint-Raviart and we adapt it for a nonconforming velocity field. We ap-

$$
\begin{aligned}
& \text { proach } \int_{\Omega} \boldsymbol{u} \cdot \nabla \boldsymbol{\tau}: \boldsymbol{\sigma} d x \text { by: } \\
& c_{1}\left(\boldsymbol{u}_{h}, \boldsymbol{\tau}_{h} ; \boldsymbol{\sigma}_{h}\right)=\sum_{\boldsymbol{e} \in \varepsilon_{h}} \int_{e} F\left(\boldsymbol{\tau}_{h}, \boldsymbol{u}_{h}, \boldsymbol{n}\right)\left[\boldsymbol{\sigma}_{h}\right] d s,
\end{aligned}
$$

where $F\left(\boldsymbol{\tau}_{h}, \boldsymbol{n}\right)=\left(\pi_{0} \boldsymbol{v}_{h} \cdot \boldsymbol{n}\right)^{+} \boldsymbol{\tau}_{h}^{\mathrm{in}}+\left(\pi_{0} \boldsymbol{v}_{h} \cdot \boldsymbol{n}\right)^{-} \boldsymbol{\tau}_{h}^{\mathrm{ext}}$

This can also be written, denoting by $\partial T^{-}=$ $\left\{e \subset \partial T ; \pi_{0}\left(\boldsymbol{u}_{h} \cdot \boldsymbol{n}\right)<0\right.$ on $\left.e\right\}$ the set of inflow boundaries:

$$
\sum_{T \in \mathcal{T}_{h}} \int_{\partial T^{-}} \boldsymbol{u}_{h} \cdot \boldsymbol{n} \tau_{h}^{\mathrm{ext}}:\left(\boldsymbol{\sigma}_{h}^{\mathrm{int}}-\boldsymbol{\sigma}_{h}^{\mathrm{ext}}\right) d s .
$$

This term requires inflow boundary conditions on the stress tensor.

The form $d_{0}(\cdot, \cdot)$ is defined as:

$$
d_{0}\left(\sigma_{h}, \boldsymbol{\tau}_{h}\right)=\sum_{T \in \mathcal{T}_{h}} \int_{T} \sigma_{h}: \tau_{h} d x
$$

and $d_{1}(\cdot, \cdot ; \cdot)$ takes into account the quadratic term:

$$
d_{1}\left(\boldsymbol{\tau}_{h}, \boldsymbol{\tau}_{h} ; \boldsymbol{\sigma}_{h}\right)=\frac{1}{2 G} \sum_{T \in \mathcal{T}_{h}} \int_{T} \tau_{h} \cdot \tau_{h}: \sigma_{h} d x .
$$

Finally, the right hand-side terms are defined as:

$$
f\left(\boldsymbol{v}_{h}\right)=\sum_{T \in \mathcal{T}_{h}} \int_{T} \boldsymbol{f} \cdot \boldsymbol{v}_{h} d x
$$

where $f \in L^{2}(\Omega)$ is a data of the problem, and:

$$
l\left(\boldsymbol{\sigma}_{h}\right)=-\sum_{e \in \varepsilon_{h}^{\partial} \cap \partial \Omega^{-}} \int_{e}\left(\boldsymbol{u}_{h} \cdot \boldsymbol{n}\right)^{-} \boldsymbol{\tau}^{D}: \boldsymbol{\sigma}_{h} d s,
$$

with $\tau^{D}$ the boundary condition.

\subsection{Quadrilateral case}

We now consider quadrilaterals meshes. To approximate the velocity and the pressure, we use RannacherTurek finite elements [34]. The degrees of freedom for the velocity are the mean values across the edges. For the pressure and the stress tensor the degrees of freedom are the same as in the triangular case (Fig. 2). This numerical scheme has also been studied in [5]. Let's recall the definition of the Rannacher-Tureck approximation space. Let $\hat{K}=[-1,1] \times[-1,1], \Psi_{K}$ : $\hat{K} \rightarrow K$ the bilinear one-to-one transformation and $\hat{Q}_{1}^{r o t}=\operatorname{span}\left\{1, \hat{x}, \hat{y}, \hat{x}^{2}-\hat{y}^{2}\right\}$. Then we define the space $Q_{K}=\left\{v ; v \circ \Psi_{K} \in \hat{Q}_{1}^{r o t}\right\}$ and we introduce the discrete spaces:

$$
\begin{aligned}
& \boldsymbol{W}_{h}=\left\{\boldsymbol{v}_{h} \in\left(L^{2}(\Omega)\right)^{2} ; \boldsymbol{v}_{h \mid K} \in\left(Q_{K}\right)^{2} \forall K \in \mathcal{K}_{h},\right. \\
& \left.\frac{1}{|e|} \int_{e}\left[v_{h}\right] d s=0 \quad \forall e \in \varepsilon_{h}^{i n t}\right\}, \\
& \boldsymbol{W}_{h}^{\boldsymbol{g}}=\left\{\boldsymbol{v}_{h} \in \boldsymbol{W}_{h} ; \int_{e} \boldsymbol{v}_{h} d s=\int_{e} \boldsymbol{g} d s \quad \forall e \in \varepsilon_{h}^{\partial}\right\}, \\
& \begin{array}{l}
\bullet \text { nodes } \\
\times \quad \text { dof for u for } \mathrm{p} \text { and } \tau
\end{array}
\end{aligned}
$$

Figure 2: Degrees of freedom for quadrilateral case.

The discrete formulation reads as follows:

$$
\left\{\begin{array}{cccc}
\left(\boldsymbol{u}_{h}, p_{h}, \boldsymbol{\tau}_{h}\right) \in \boldsymbol{W}_{h}^{\boldsymbol{g}} \times Q_{h} \times \boldsymbol{X}_{h} & & & \\
a\left(\boldsymbol{u}_{h}, \boldsymbol{u}_{h} ; \boldsymbol{v}_{h}\right)+b\left(p_{h}, \boldsymbol{v}_{h}\right) & & & \\
\quad+c_{0}\left(\boldsymbol{v}_{h}, \boldsymbol{\tau}_{h}\right) & = & f\left(\boldsymbol{v}_{h}\right) & \forall \boldsymbol{v}_{h} \in \boldsymbol{W}_{h}^{\mathbf{0}} \\
b\left(q_{h}, \boldsymbol{u}_{h}\right)+ & = & \forall q_{h} \in Q_{h} \\
c\left(\boldsymbol{u}_{h}, \boldsymbol{\tau}_{h} ; \boldsymbol{\sigma}_{h}\right)+d\left(\boldsymbol{\tau}_{h}, \boldsymbol{\tau}_{h} ; \boldsymbol{\sigma}_{h}\right) & =l\left(\boldsymbol{\sigma}_{h}\right) & \forall \boldsymbol{\sigma}_{h} \in \boldsymbol{X}_{h}
\end{array}\right.
$$

The main difference with the triangular case is the addition of a regularization term. The form $a(., . ;$.$) is$ now decomposed into three parts:

$a\left(\boldsymbol{u}_{h}, \boldsymbol{u}_{h} ; \boldsymbol{v}_{h}\right)=a_{0}\left(\boldsymbol{u}_{h}, \boldsymbol{u}_{h} ; \boldsymbol{v}_{h}\right)+\gamma a_{1}\left(\boldsymbol{u}_{h}, \boldsymbol{v}_{h}\right)+2 R\left(\boldsymbol{u}_{h}, \boldsymbol{v}_{h}\right)$. 
The $R(.,$.$) term is added to ensure the discrete coer-$ civity and is given by :

$$
R\left(\boldsymbol{u}_{h}, \boldsymbol{v}_{h}\right)=\eta \sum_{K \in \mathcal{K}_{h}} \int_{K}\left(\boldsymbol{D}\left(\boldsymbol{u}_{h}\right)-\pi_{0}^{K} \boldsymbol{D}\left(\boldsymbol{u}_{h}\right)\right): \boldsymbol{D}\left(\boldsymbol{v}_{h}\right) d x .
$$

Remark 1. The analysis of both triangular and quadrangular cases have been performed for the underlying Stokes problem. We showed the well-posedness of the stabilized formulations and we have obtained optimal a priori error estimates. For more details, one may refers to [5].

\section{Results and discussion}

In this section, we present numerical simulations obtained with triangular meshes (channel and 4:1 abrupt contraction) and quadrilateral meshes (cylinder). We use structured meshes.

\subsection{Solvers}

The non linear problems are solved by means of Newton's method. At each Newton's iteration, a linear problem has to be solved. Depending on the geometry and on the mesh type (triangular or quadrilateral), two linear solvers have been used: a direct solver and a multigrid approach. Indeed, the quadrilateral case is more costly. In this case, the use of a multigrid method based on Vanka's smoother allows us to deal with fine meshes.

Remark 2. The Newton's method necessitates the computation of the following Jacobian matrix:

$$
\left(\begin{array}{ccc}
A_{0, \boldsymbol{u}}+\gamma A_{1}+2 R & B & C_{0} \\
B^{t} & 0 & 0 \\
-2 \eta C_{0}^{t}+C_{1, \boldsymbol{u}}+C_{2, \boldsymbol{u}} & 0 & D_{0}+C_{1, \tau}+C_{2, \tau}+\alpha D_{1, \tau}
\end{array}\right) .
$$

Let $\boldsymbol{u}^{i}$ and $\boldsymbol{\tau}^{i}$ be the solution computed at the previous Newton iterate, the corresponding forms are defined as follows:

$$
\begin{aligned}
a_{0, \boldsymbol{u}}\left(\boldsymbol{u}_{h}, \boldsymbol{v}_{h}\right) & =\sum_{T \in \mathcal{T}_{h}} \int_{T}\left(\left(\boldsymbol{u}^{i} \cdot \nabla\right) \boldsymbol{u}_{h} \boldsymbol{v}_{h}+\left(\boldsymbol{u}_{h} \cdot \nabla\right) \boldsymbol{u}^{i} \boldsymbol{v}_{h}\right) d x, \\
c_{1, \boldsymbol{u}}\left(\boldsymbol{u}_{h}, \boldsymbol{\sigma}_{h}\right) & =\sum_{T \in \mathcal{T}_{h}} \int_{\partial T^{-}} \boldsymbol{u}_{h} \cdot \boldsymbol{n} \boldsymbol{\tau}^{e x t}:\left(\boldsymbol{\sigma}_{h}^{i n t}-\boldsymbol{\sigma}_{h}^{e x t}\right) d s \\
c_{1, \tau}\left(\boldsymbol{\tau}_{h}, \boldsymbol{\sigma}_{h}\right) & =\sum_{T \in \mathcal{T}_{h}} \int_{\partial T^{-}} \boldsymbol{u}^{i} \cdot \boldsymbol{n} \tau_{h}^{e x t}:\left(\boldsymbol{\sigma}_{h}^{\text {int }}-\boldsymbol{\sigma}_{h}^{e x t}\right) d s
\end{aligned}
$$

$$
\begin{aligned}
& c_{2, \boldsymbol{u}}\left(\boldsymbol{u}_{h}, \boldsymbol{\sigma}_{h}\right)=-\lambda \sum_{T \in \mathcal{T}_{h}} \int_{T} \boldsymbol{\tau}^{i} \nabla \boldsymbol{u}_{h}: \boldsymbol{\sigma}_{h} d x \\
&-\lambda \sum_{T \in \mathcal{T}_{h}} \int_{T}\left(\boldsymbol{\nabla} \boldsymbol{u}_{h}\right)^{t} \boldsymbol{\tau}^{i}: \boldsymbol{\sigma}_{h} d x, \\
& c_{2, \tau}\left(\boldsymbol{\tau}_{h}, \boldsymbol{\sigma}_{h}\right)=-\lambda \sum_{T \in \mathcal{T}_{h}} \int_{T} \boldsymbol{\tau}_{h} \boldsymbol{\nabla} \boldsymbol{u}^{i}: \boldsymbol{\sigma}_{h} d x \\
&-\lambda \sum_{T \in \mathcal{T}_{h}} \int_{T}\left(\boldsymbol{\nabla} \boldsymbol{u}^{i}\right)^{t} \boldsymbol{\tau}_{h}: \boldsymbol{\sigma}_{h} d x, \\
& d_{1, \tau}\left(\boldsymbol{\tau}_{h}, \boldsymbol{\sigma}_{h}\right)=\sum_{T \in \mathcal{T}_{h}} \frac{1}{2 G} \int_{T}\left(\boldsymbol{\tau}_{h} \boldsymbol{\tau}^{i}+\boldsymbol{\tau}^{i} \boldsymbol{\tau}_{h}\right): \boldsymbol{\sigma}_{h} d x .
\end{aligned}
$$

\subsection{Implementation of the model}

Let's recall that our main goal is to obtain realistic results for high Weissenberg numbers. The Newton method does not converge if we consider directly a large Weissenberg number. To avoid this problem, we consider an evolution method. The code starts the calculation using an existing results file computed with a smaller value of $\lambda$ as an initial solution. At each step, the $\lambda$ gap is fitted to obtain the convergence of the Newton method.

\subsection{Mesh convergence}

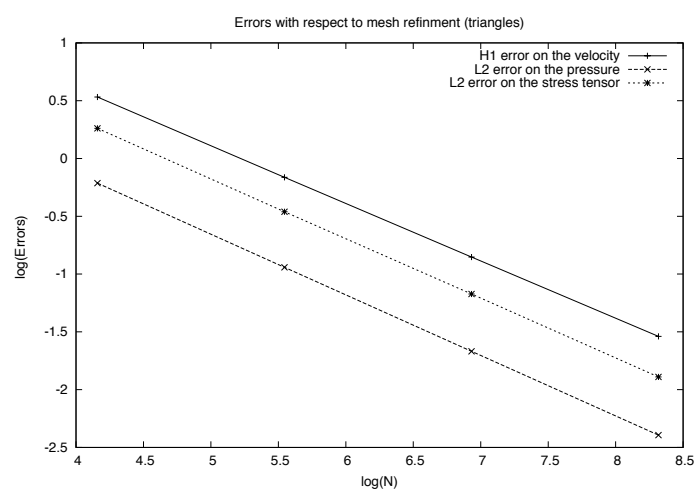

Figure 3: Mesh convergence, triangular case.

No exact solution exists for the Giesekus model. To check the convergence of our method with respect to mesh refinement, we consider the Giesekus model with right-hand side such that the solution on $\Omega=[-1,1] \times$ $[-1,1]$ with Dirichlet boundary condition is equal to:

$\boldsymbol{u}=\left(\begin{array}{c}1+x+2 x^{2}+2 y^{2}+x y \\ 1-y+2 x^{2}-\frac{1}{2} y^{2}-4 x y\end{array}\right), p=x-y, \boldsymbol{\tau}$ constant. 
Results are presented in Fig. 3 for the triangular case and in Fig. 4 for the quadrilateral case. As expected we obtain the optimal convergence rate: $O\left(N^{-1 / 2}\right)$, with $N$ the number of elements. Note that similar results can be found in [5] for a linear solution for $\tau$.

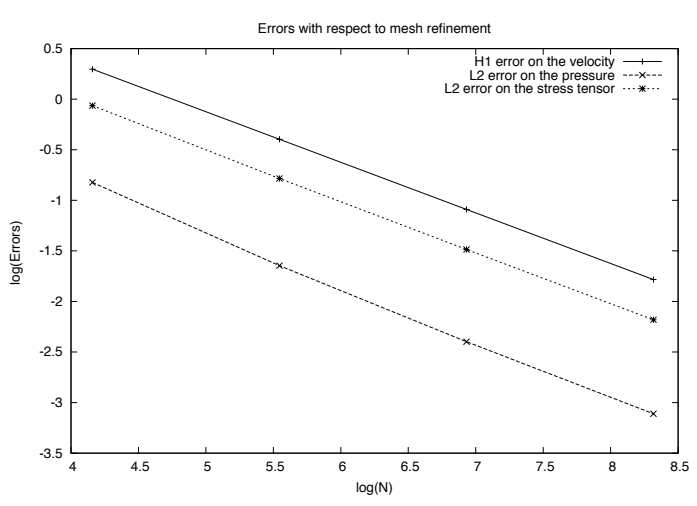

Figure 4: Mesh convergence, quadrilateral case.

\subsection{Simulation vs. analytical solutions}

We consider the flow of an affine Phan-Thien and Tanner liquid with $\epsilon=0.05$ and a Giesekus liquid with $\alpha=0.5$ along a channel. To validate our approach, we compare the computed velocity profile and the analytical solutions for the fully developed channel flow. For reasons of symmetry, only the lower part of the geometry is taken as a flow domain. The dimensional characteristics and the definition of boundaries of these geometries are given in Fig. 5 and we take $a=1 \mathrm{~mm}$.

The boundary conditions are defined as follows:

- Inflow on $\Gamma_{1}$, flat velocity profile typically $0.1 \mathrm{~m} / \mathrm{s}$.

- Homogeneous Dirichlet boundary conditions on $\Gamma_{2}$.

- Outflow, Neumann boundary condition on $\Gamma_{3}$.

- Symmetry plane on $\Gamma_{4}$.

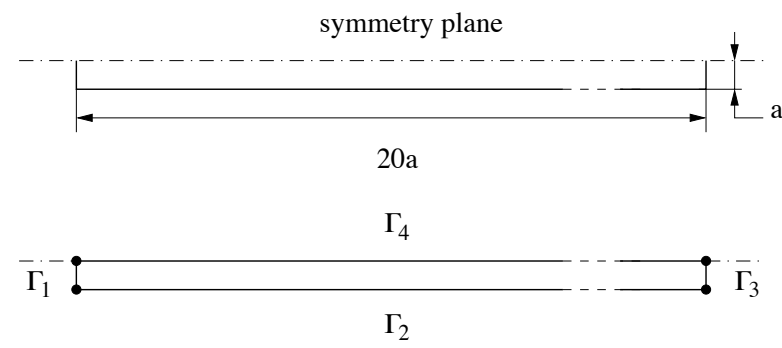

Figure 5: Channel geometry.
Several definitions of the Weissenberg number have been proposed $[25,38]$. In this work, we adopt the following definition:

$$
\mathfrak{W i}=\lambda \dot{\gamma}=\lambda \frac{3 \bar{u}}{a}
$$

where $\bar{u}$ is the inflow velocity or the average velocity on the channel. The shear rate is calculated on the wall for the equivalent Newtonian liquid.

For the affine Phan-Thien and Tanner model, the velocity profile is given by the following relationship [27]:

$$
\begin{aligned}
u_{x}(y)=-\frac{a^{2}}{2 \eta}\left(1-\frac{y^{2}}{a^{2}}\right) & \\
& \left(1+\frac{\epsilon \lambda^{2}}{\eta^{2}} a^{2}\left(1+\frac{y^{2}}{a^{2}}\right)\left(\frac{\partial p}{\partial x}\right)^{2}\right) \frac{\partial p}{\partial x}
\end{aligned}
$$

and the average velocity by:

$$
\bar{u}=-\frac{a^{2}}{3 \eta}\left(1+\frac{6}{5} \frac{\epsilon \lambda^{2}}{\eta^{2}} a^{2}\left(\frac{\partial p}{\partial x}\right)^{2}\right) \frac{\partial p}{\partial x}
$$

The Weissenberg number is given by:

$$
\mathfrak{W} i=-\frac{\lambda a}{\eta}\left(1+\frac{6}{5} \frac{\epsilon \lambda^{2}}{\eta^{2}} a^{2}\left(\frac{\partial p}{\partial x}\right)^{2}\right) \frac{\partial p}{\partial x}
$$

The pressure gradient is the real solution of the cubic equations (16) or (17).

In the case of the Giesekus liquid, an analytical solution is given in [25]:

$$
u_{x}(y)=\frac{1}{2 \beta \lambda} \ln \frac{1-a^{2} \beta^{2}}{1-y^{2} \beta^{2}}, \quad \beta=\frac{\lambda}{\eta} \frac{\partial p}{\partial x}
$$

and the average velocity by:

$$
\bar{u}=\frac{1}{\beta \lambda}\left(1-\frac{\operatorname{atanh}(a \beta)}{a \beta}\right)
$$

$\beta$ is related to the Weissenberg number by:

$$
\mathfrak{W} \mathfrak{i}=\frac{3}{2 a \beta}\left(1-\frac{\operatorname{atanh}(a \beta)}{a \beta}\right)
$$

The characteristics of the liquid chosen for all the simulations are $10^{3}$ Pa.s for the viscosity and $10^{3} \mathrm{~kg} / \mathrm{m}^{3}$ for the density. The inlet flow is equal to $0.1 \mathrm{~m} / \mathrm{s}$. These conditions give $10^{-4}$ as Reynolds number and $300 \lambda$ as Weissenberg number. With this geometry, we employ a mesh consisting of 40960 elements. 


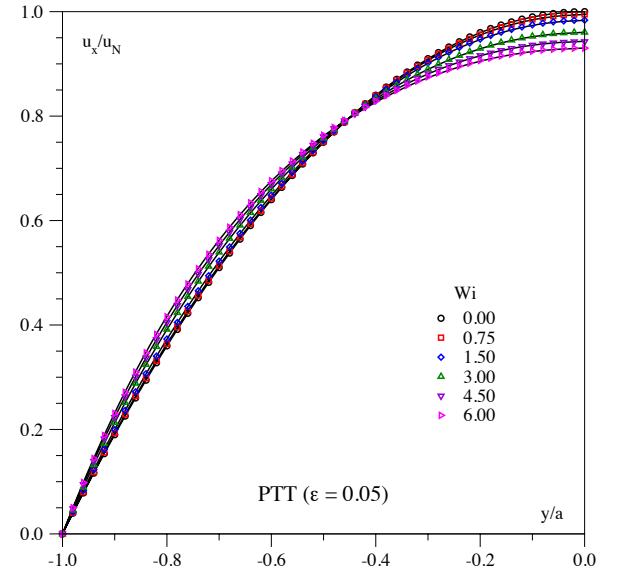

Figure 6: Profiles of the dimensionless velocity $u_{x} / u_{N}$ vs. $y / a$ with $u_{N}$ the Newtonian velocity. 2D planar flow in a channel for a Phan-ThienTanner liquid, $\epsilon=0.05$. Comparison between numerical (symbol) and analytical (line) solutions.

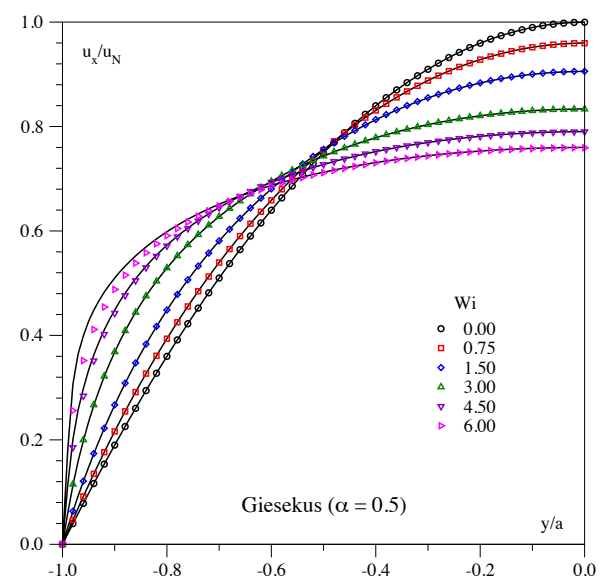

Figure 7: Profiles of the dimensionless velocity $u_{x} / u_{N}$ vs. $y / a$ with $u_{N}$ the Newtonian velocity. 2D planar flow in a channel for a Giesekus liquid, $\alpha=0.5$. Comparison between numerical (symbol) and analytical (line) solutions.

The comparison between numerical and analytical profiles is given in Figures 6 and 7. The velocity profiles obtained are in good accordance.

In the case of the Phan-Thien-Tanner liquid, the velocity profiles are directly calculated from the equations (15) and (17). For the Giesekus liquid, the parameter $\beta$ is determined from the Weissenberg number (20). With this value and the velocity equation (18), we calculate the velocity profile.

\subsection{Drag comparison}

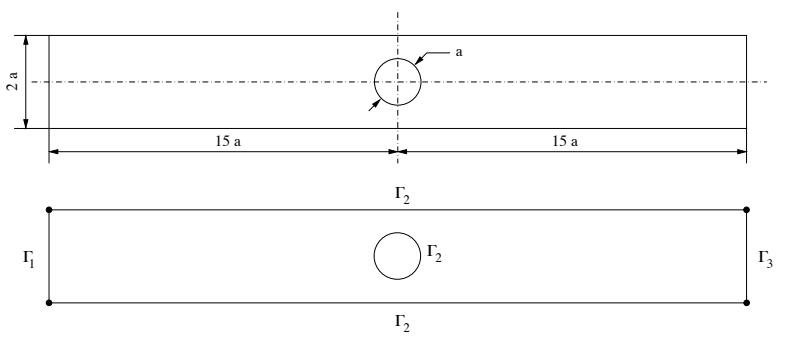

Figure 8: Geometry for a flow past a cylinder.

Now, we consider the flow past a cylinder benchmark used in $[10,29,18,11,8]$. The geometry is given in Figure 8. We impose the same inflow conditions as in [8], a parabolic velocity profile with $\bar{u}=1 \mathrm{~m} / \mathrm{s}$. On the outflow we impose a homogeneous Neumann condition, and on the other boundaries, including the cylinder, noslip conditions.

On this benchmark, most of the drag results are presented for the Oldroyd-B model. To compare our schemes with other numerical approaches, we have considered this rheological model.

As in the cited papers, we take $\eta=\eta_{n}+\eta_{p}=1$, $\eta_{p}=0.41$ and the Weissenberg number defined by $\mathfrak{B i}=$ $\lambda \bar{u} / a$, with $\bar{u}$ the inflow mean velocity.

The drag along the cylinder $\Gamma_{c}$ is given by the relationship:

$$
\mathcal{D}=\int_{\Gamma_{c}}(1,0)^{T} \cdot \boldsymbol{\Pi} \boldsymbol{n} d s
$$

with $\boldsymbol{\Pi}=\boldsymbol{\tau}_{p}-p \boldsymbol{I}+2 \eta_{n} \boldsymbol{D}$ the total stress tensor.

We have implemented the same numerical scheme as for the Giesekus model. In order to obtain accurate drag values, the meshes are more refined around the cylinder, as presented in Fig. 9.

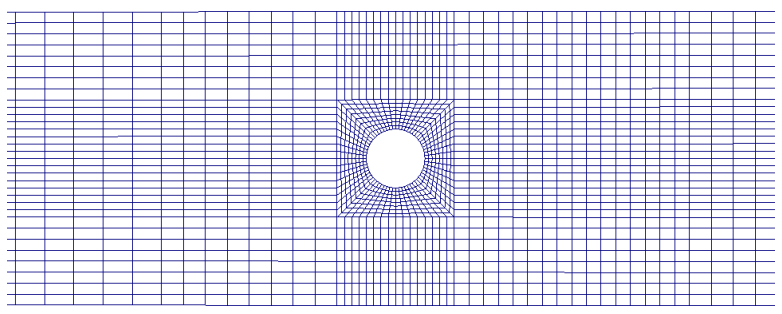

Figure 9: Mesh shape for the drag calculation.

In this case the multigrid method allows us to use meshes composed by up to 1048576 elements. We have then computed in Tab.1 the drag values in term of 
the number of elements $N$ for $\lambda=0.6$. The linear convergence obtained leads to more accurate extrapolated values $\mathcal{D}^{*} . n_{N}$ denotes the number of Newton iterates whereas $n_{M}$ is the sum of the multigrid iterates.

\begin{tabular}{|r|c|c|c|c|c|}
\hline$N$ & $n_{N}$ & $n_{M}$ & $\mathcal{D}$ & $\Delta \mathcal{D}$ & $\mathcal{D}^{*}$ \\
\hline 1024 & 7 & 19 & 118.081 & - & - \\
4096 & 6 & 12 & 118.421 & 0.340 & - \\
16384 & 6 & 18 & 118.349 & 0.072 & - \\
65536 & 6 & 24 & 118.085 & 0.264 & 117.821 \\
262144 & 5 & 20 & 117.936 & 0.149 & 117.787 \\
1048576 & 5 & 31 & 117.858 & 0.078 & 117.780 \\
\hline
\end{tabular}

Table 1: Drag values for $\lambda=0.6$.

One may see in Tab. 2 that the drag values $\mathcal{D}$ obtained with Concha on a mesh consisting of 1048576 elements for different $\lambda$ are quite close to those of the literature, in particular with [8] and [18].

\begin{tabular}{|c||c|c|c|c|}
\hline$\lambda$ & 0.0 & 0.3 & 0.6 & 0.7 \\
\hline \hline Concha & 132.357 & 123.190 & 117.780 & 117.321 \\
Ref. [10] & 131.809 & 123.514 & 120.485 & - \\
Ref. [29] & 132.357 & - & 117.775 & - \\
Ref. [18] & 132.358 & 123.193 & 117.792 & 117.290 \\
Ref. [11] & 132.330 & 123.410 & - & - \\
Ref. [8] & - & 123.194 & 117.779 & 117.321 \\
\hline
\end{tabular}

Table 2: Comparison of drag values with the literature.

\subsection{Simulation vs. experimental data}

In 1994, Quinzani et al. [32] measured the detailed flow fields of a well characterised polymer solution flowing in a planar abrupt contraction by using LaserDoppler velocimetry (LDV) and flow-induced birefringence (FIB). We used these experimental results to validate our code. A mesh of 32768 elements was used for these simulations.

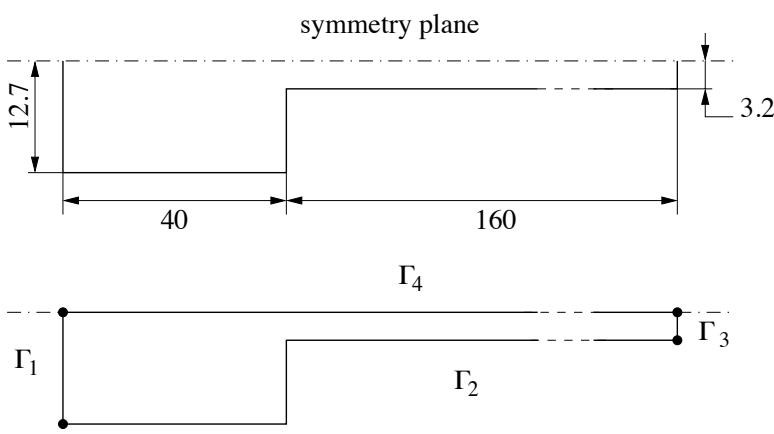

Figure 10: 4:1 abrupt contraction geometry from [32] (mm).
Their fluid test is a solution of a high molecule weight polyisobutylene (PIB) dissolved in tetradecane $\left(\mathrm{C}_{14} \mathrm{H}_{30}\right)$. The material parameters of the solution are: $\eta_{0}=1.24$ Pa.s and $\lambda=0.06 \mathrm{~s}$ at $25^{\circ} \mathrm{C}$. The density of this fluid is $800 \mathrm{~kg} / \mathrm{m}^{3}$. The test section consists of two removable inserts fitted into an outer shell with $5.1 \mathrm{~cm}$ height, $25.4 \mathrm{~cm}$ width, and $51 \mathrm{~cm}$ length. The inserts form a planar contraction of upstream thickness $2.54 \mathrm{~cm}$ and downstream thickness $0.64 \mathrm{~cm}$ to give a contraction ratio of 3.97:1 (Fig. 10). The aspect ratio of the flow cell is smallest upstream of the contraction plane, where it is 10:1. According to Xue [38], the 2D flow simulation is only a good approximation to the fully $3 \mathrm{D}$ flow if the upstream aspect ratio in the experiment is at least 10 . The average velocity in the small channel is $2.14 \mathrm{~cm} / \mathrm{s}$ corresponding to $0.539 \mathrm{~cm} / \mathrm{s}$ for the inflow velocity. All experimental data have been scanned from the paper of Quinzani et al. [32].

Quinzani et al. define the Weissenberg number by the following relationship:

$$
\mathfrak{W i}=\lambda(\dot{\gamma}) \dot{\gamma}=\lambda(\dot{\gamma}) \frac{\bar{u}}{h}
$$

where $\bar{u}$ is the average velocity in the small channel and $h$ its half height. They obtain $\mathfrak{B} \mathfrak{i}=0.25$.

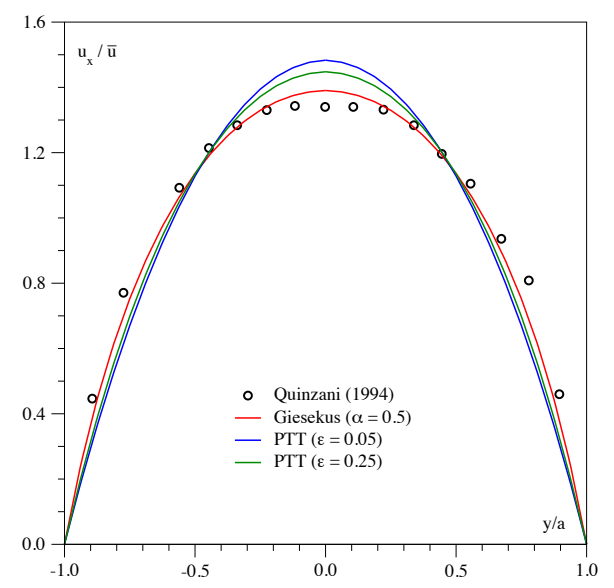

Figure 11: Profiles of the dimensionless velocity $u_{x} / \bar{u}$ vs. $y / a$. 2D planar flow in a 3.97:1 contraction for a Phan-Thien-Tanner liquid, $\epsilon=0.05,0.25$ and a Giesekus liquid, $\alpha=0.5$. Comparison between numerical solutions and experimental data (data scanned from Quinzani [32]).

In Figure 11, we compare the dimensionless velocity profile for Phan-Thien-Tanner and Giesekus liquids with the experimental data. The numerical results for the Giesekus liquid are in very good agreement with 
experimental observation. For the Phan-Thien-Tanner model, the maximum velocities are overestimated.

The velocity component $u_{x}$ in the downstream and the upstream channels is given in the Figure 12 for the results of simulation with the Giesekus liquid and the experimental data. The dimensionless cordinate $\xi$ is defined as $\left(x-x_{0}\right) / h$ where $x_{0}$ is the $x$-coordinate of the contraction $\left(x_{0}=40 \mathrm{~mm}\right)$ and $h$ is the half-width of the small channel $(h=3.2 \mathrm{~mm})$. The agreement is globally good.
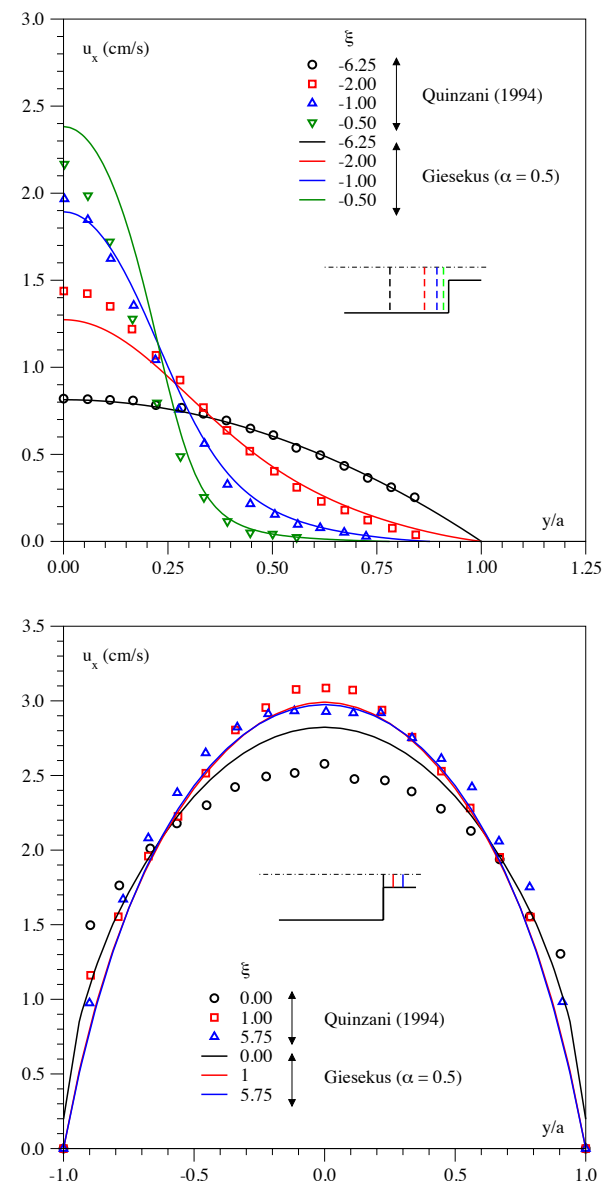

Figure 12: Profiles of velocity $u_{x}$ vs. $y / a$. 2D planar flow in a 3.97:1 contraction for a Giesekus liquid, $\alpha=0.5$. Comparison between numerical solutions and experimental data in the downstream and the upstream channels (data scanned from Quinzani [32]).

For the upstream channel, we observe a gap between the numerical results and the experimental data near the central part of the flow. The deviations may be attributed to the experimental errors. Nevertheless the numerical results are in good agreement with experiment.
For the downstream channel, we observe clearly a difference between numerical results and experimental data on the abrupt contraction plane $(\xi=0)$. Again, we suggest that this difference is due to experimental errors. Near the contraction, the velocity profile is particularly sensitive to the $x$ position. The difference between the maximum velocities for $\xi=0$ and $\xi=-0.15$, i.e. $0.5 \mathrm{~mm}$, is approximately $4 \%$. The measuring volumes formed from the beams of laser (LDV) are ellipsoids with dimensions of approximately $50 \mu \mathrm{m} \times 50 \mu \mathrm{m} \times 250 \mu \mathrm{m}$.
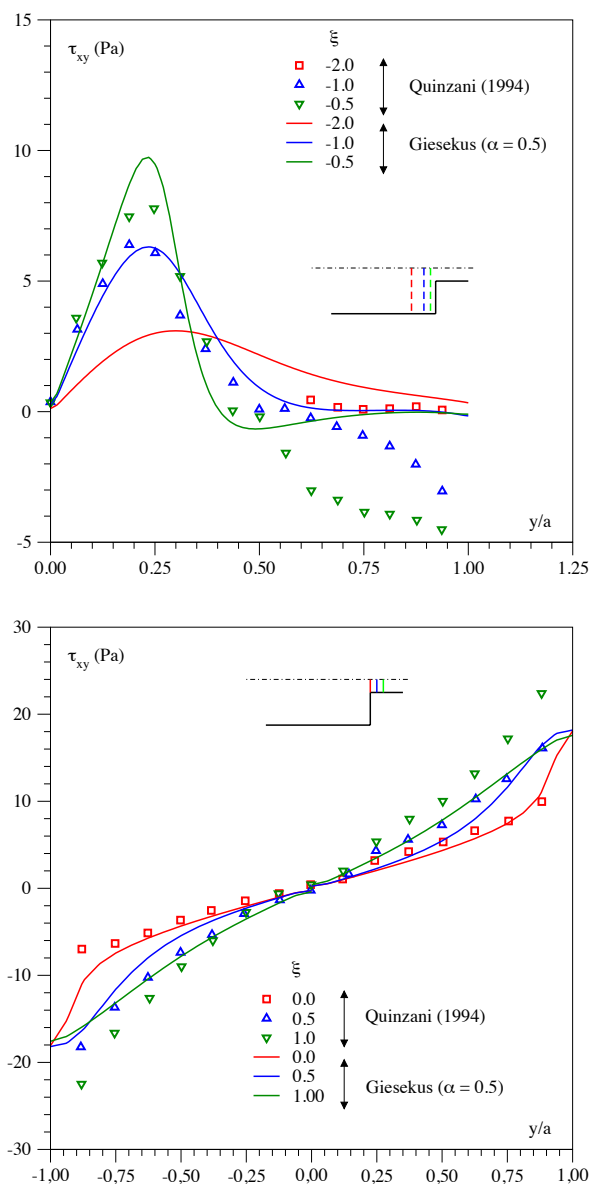

Figure 13: Profiles of shear stress $\tau_{x y}$ vs. $y / a$. 2D planar flow in a 3.97: 1 contraction for a Giesekus liquid, $\alpha=0.5$. Comparison between numerical solutions and experimental data in the downstream and the upstream channels (data scanned from Quinzani [32]).

The shear stress $\tau_{x y}$ and normal stress difference $\left(\tau_{x x}-\tau_{y y}\right)$ in the downstream and the upstream channels are given in the Figures 13 and 14. Our simulations are in qualitative agreement with the experiments. 
In the upstream channel, our simulations give a suitable description of the flow for $0<y / a<0.5$ for the shear and the normal stresses. In the recirculation zone, we observe a disagreement between the computed results and the experimental data. The Figures 15 shows the position of dimensionless coordinate $\xi$ compared with the corner vortex size of our simulation. The disagreement is significant in the vortex. As $\tau_{x y} \propto \dot{\gamma}$, the velocity profiles (Fig. 12) give informations about the level of the shear in the vortex. We note that the experimental shear stresses appear to be overestimated.

In the case of the downstream channel, the behaviour of the shear and normal stresses is correctly predicted by our computations. The meshes used in this simulation are too coarse to resolve the shear stress near the wall.
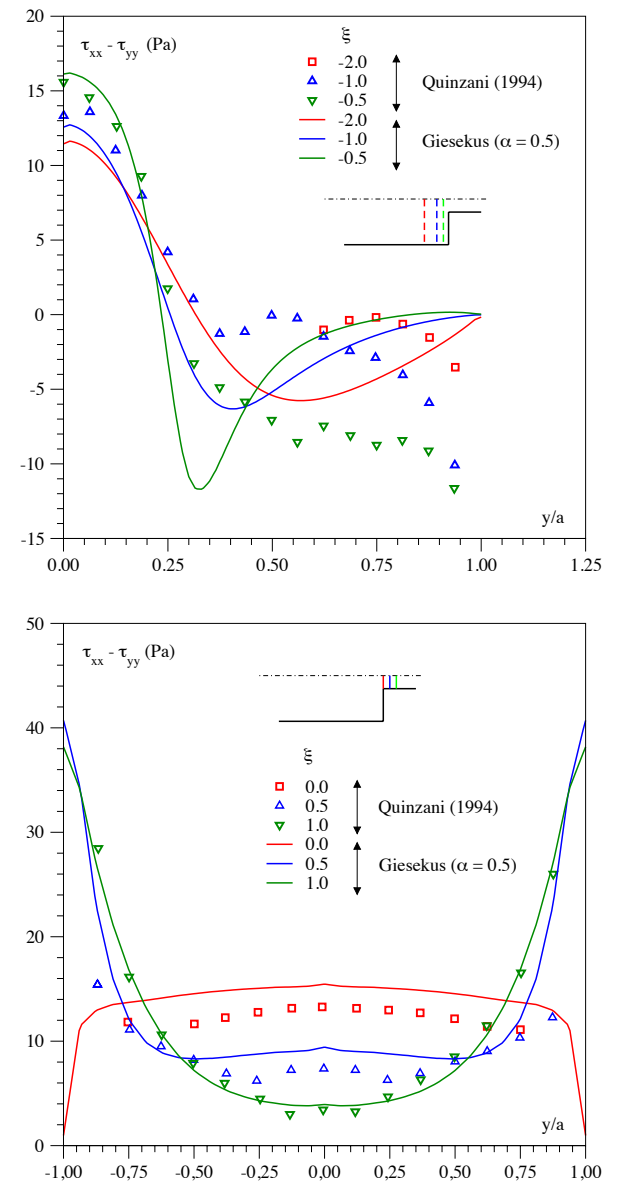

Figure 14: Profiles of normal stress $\tau_{x x}-\tau_{y y}$ vs. $y / a$. 2D planar flow in a 3.97:1 contraction for a Giesekus liquid, $\alpha=0.5$. Comparison between numerical solutions and experimental data in the downstream and the upstream channels (data scanned from Quinzani [32]).

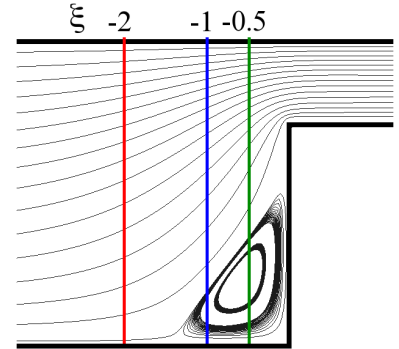

Figure 15: Streamlines in 2D planar flow in a 3.97:1 contraction for Giesekus liquid, $\alpha=0.5$.

\section{7. $4: 1$ simulations}

The 4:1 abrupt contraction geometry is a classic benchmark flow problem used in computational fluid mechanics. The dimensional characteristics and the definition of boundaries of this geometry are given in Fig. 16 and we take $a=1 \mathrm{~mm}$.

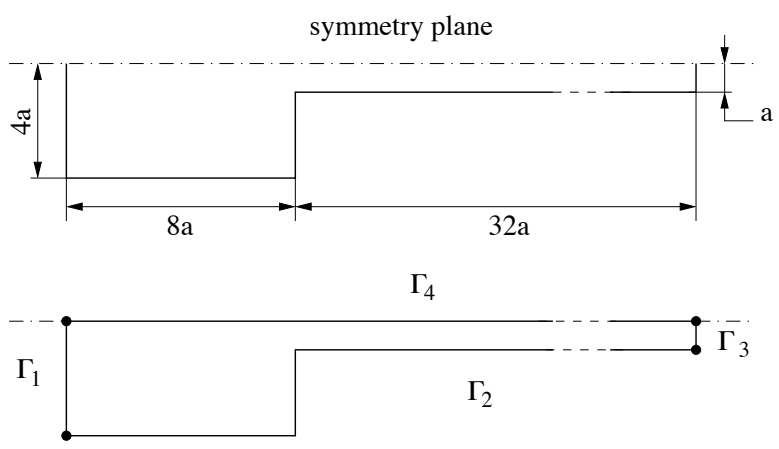

Figure 16: 4:1 abrupt contraction geometry.

Many polymeric liquids exhibit large recirculating vortices upstream of the entry on an abrupt contraction. Our numerical simulations predict this phenomenon. For the 4:1 geometry, the streamlines for each Weissenberg numbers and for affine Phan-Thien-Tanner and Giesekus $(\alpha=0.5)$ liquids are presented in Figure 17 and 18 . We observed a growth of vortices with increasing Weissenberg numbers. Lip vortices were observed only in the case of Giesekus liquid with $\alpha=0$ and for Weissenberg number values near 4.5. For this value of $\alpha$, the upper-convected Maxwell (UCM) model is recovered.

In the Figures 19, we show the numerical results of velocities along the plane of symmetry for different Weissenberg numbers. The solutions of the flow develop numerical instabilities in the form of oscillations with increasing Weissenberg number. The upper limit value of Weissenberg number is controlled by 
these oscillations. We obtain approximately 22 as critical Weissenberg number for the affine PTT liquid and the Giesekus liquid with $\alpha=0$. No oscillation was observed for the Giesekus liquid and the value of 30 for the Weissenberg number was reached.

We have obtained simulations for high Weissenberg numbers for the Giesekus model ( $\mathfrak{B i}>21)$. Two approaches might explain the quality of those results.

The first explanation is inherent to physical characteristics of rheological models. Indeed, the Weissenberg number is a measure of the elastic properties of the polymer liquid, the elasticity is also represented by the first normal-stress difference $N_{1}$. In the high shear-rate range, the variation of this material function is:

- Phan-Thien-Tanner: $N_{1}(\dot{\gamma}) \propto \dot{\gamma}^{2 / 3}$

- Giesekus $(\alpha=0)$ or UCM: $N_{1}(\dot{\gamma}) \propto \dot{\gamma}^{2}$

- Giesekus $(\alpha=0.5): N_{1}(\dot{\gamma}) \propto \dot{\gamma}^{1 / 2}$

The increase of the normal stress is smaller for the Giesekus liquid than for the affine Phan-Thien-Tanner liquid or the upper-convected Maxwell model. The likely effect of normal stress on simulation is the loss of convergence.

The normal stress $N_{1}$ along the centerline is given in Figure 20. The maximum of the normal stress is obtained after the abrupt contraction for the Phan-ThienTanner liquid or the upper-convected Maxwell model and before the abrupt contraction for the Newtonian fluid and the Giesekus liquid. The maximum values of the normal stress decreases with the Weissenberg number for the Phan-Thien-Tanner and Giesekus liquids and increases for the UCM, i.e. Giesekus with $\alpha=0$. In the last case, we show some oscillations for $\mathfrak{B i}=21$.

Secondly, the loss of convergence of the algorithms for high Weissenberg numbers is a major issue in computational rheology and is associated with the loss of the positivity of the so-called conformation tensor at the discrete level. Numerical schemes preserving this property yield energy estimates and are more stable. In [4], the authors showed that our discretization associated with the Giesekus model yields the positivity of the discrete conformation tensor. The key point for this result is the use of a DG0 approximation for the stress tensor. Indeed, the discretization of the convective term on the stress tensor (13) is crucial to ensure the positivity of the discrete stress tensor for the Giesekus equation.

\section{Conclusion}

In this work, we present numerical results obtained with a finite element approximation of the Giesekus and the PTT models. The velocity and the pressure are approximated by non-conforming finite elements of Crouzeix-Raviart in the triangular case and of Rannacher-Tureck in the quadrilateral case while the stress tensor is approximated by $P_{0}$ totally discontinuous finite elements.

We have presented comparisons with analytical solutions, with experiments and also with the literature illustrating the good behaviour of our numerical scheme. Moreover, we show the superiority of the Giesekus model, which seems to be the more appropriate to obtain realistic simulation. The nonconforming finite element method applied to this model seems to be stable for the triangular and quadrilateral cases. We have obtained realistic simulations for high Weissenberg numbers superior to 21 on the popular test-case of an abrupt 4:1 contraction.

\section{Acknowledgement}

The authors would like to thank the reviewers for their helpful comments. 


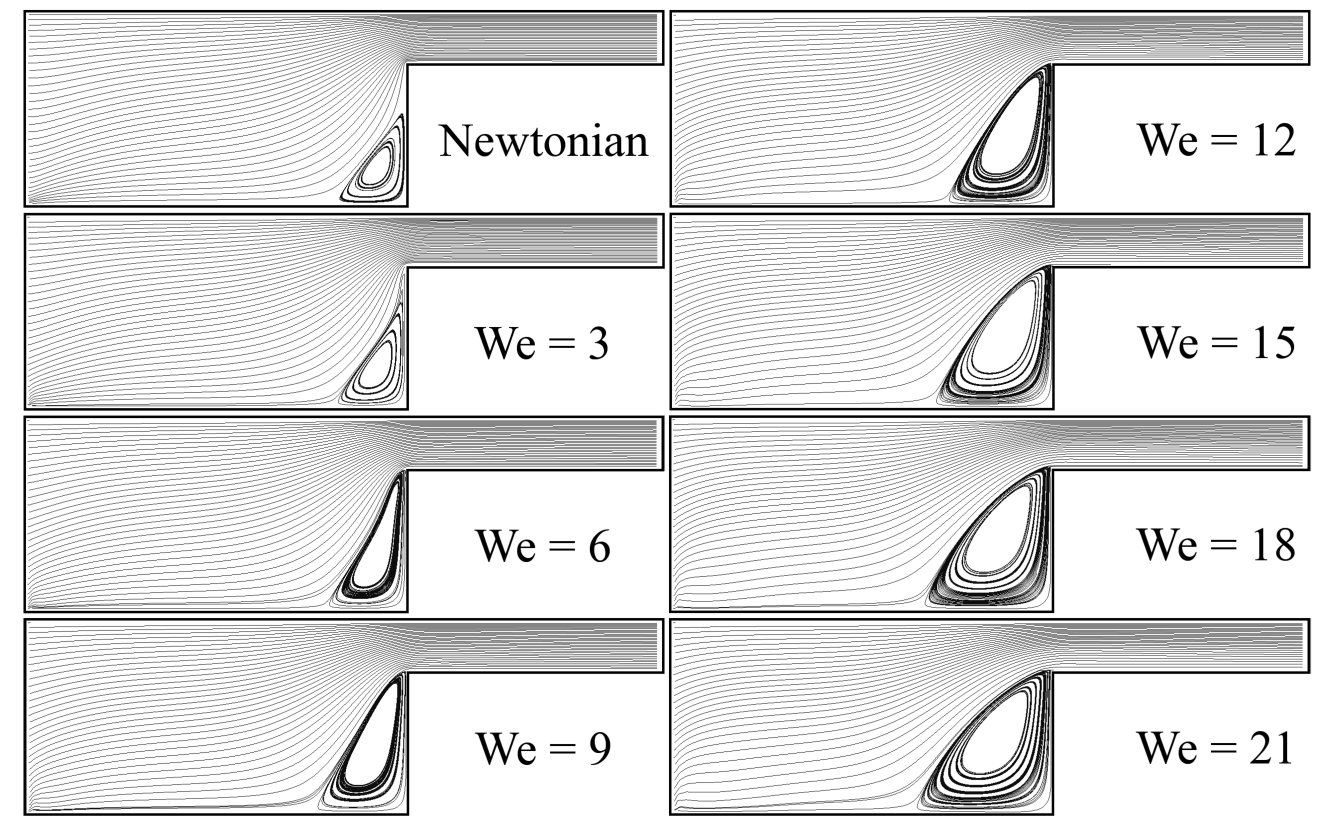

Figure 17: 4:1 contraction. Streamlines for Phan-Thien-Tanner liquid, $\epsilon=0.05$.

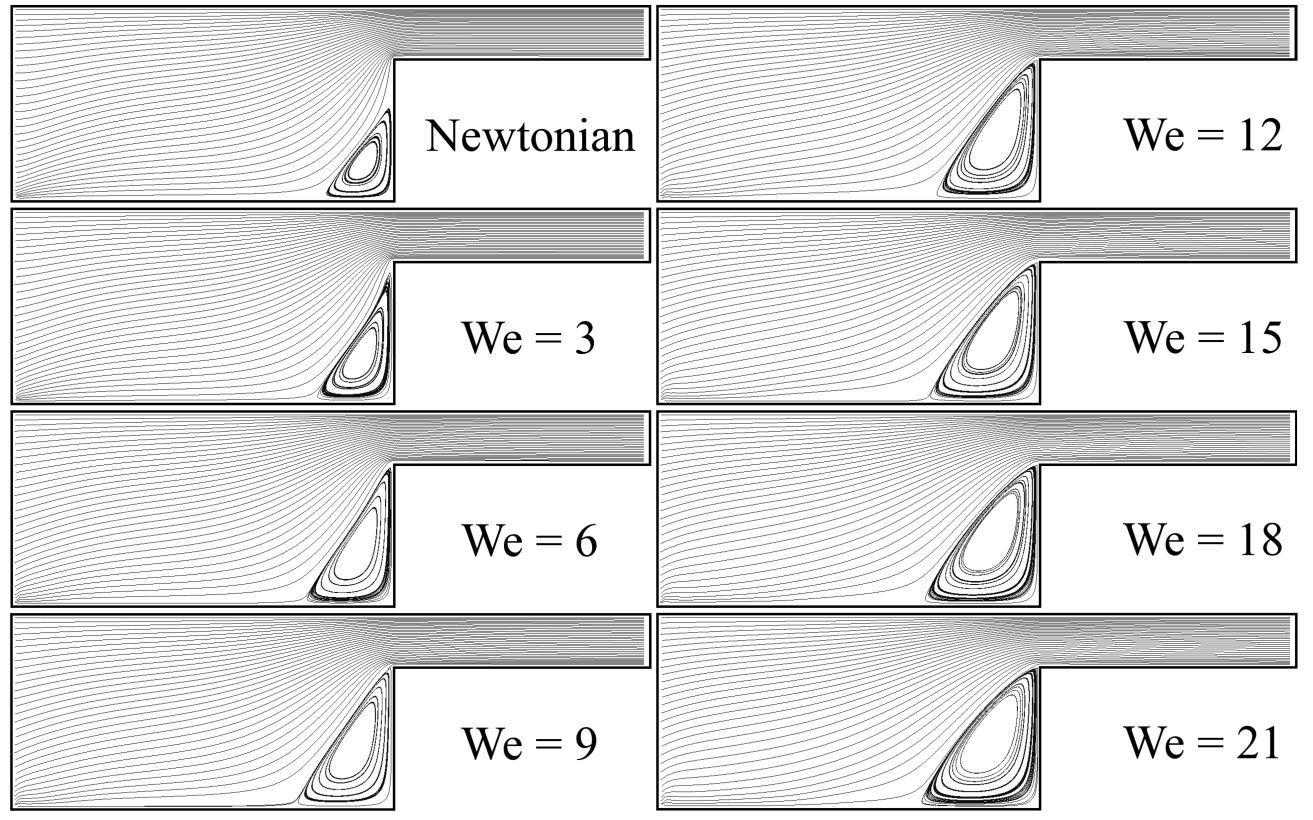

Figure 18: 4:1 contraction. Streamlines for Giesekus liquid, $\alpha=0.5$. 

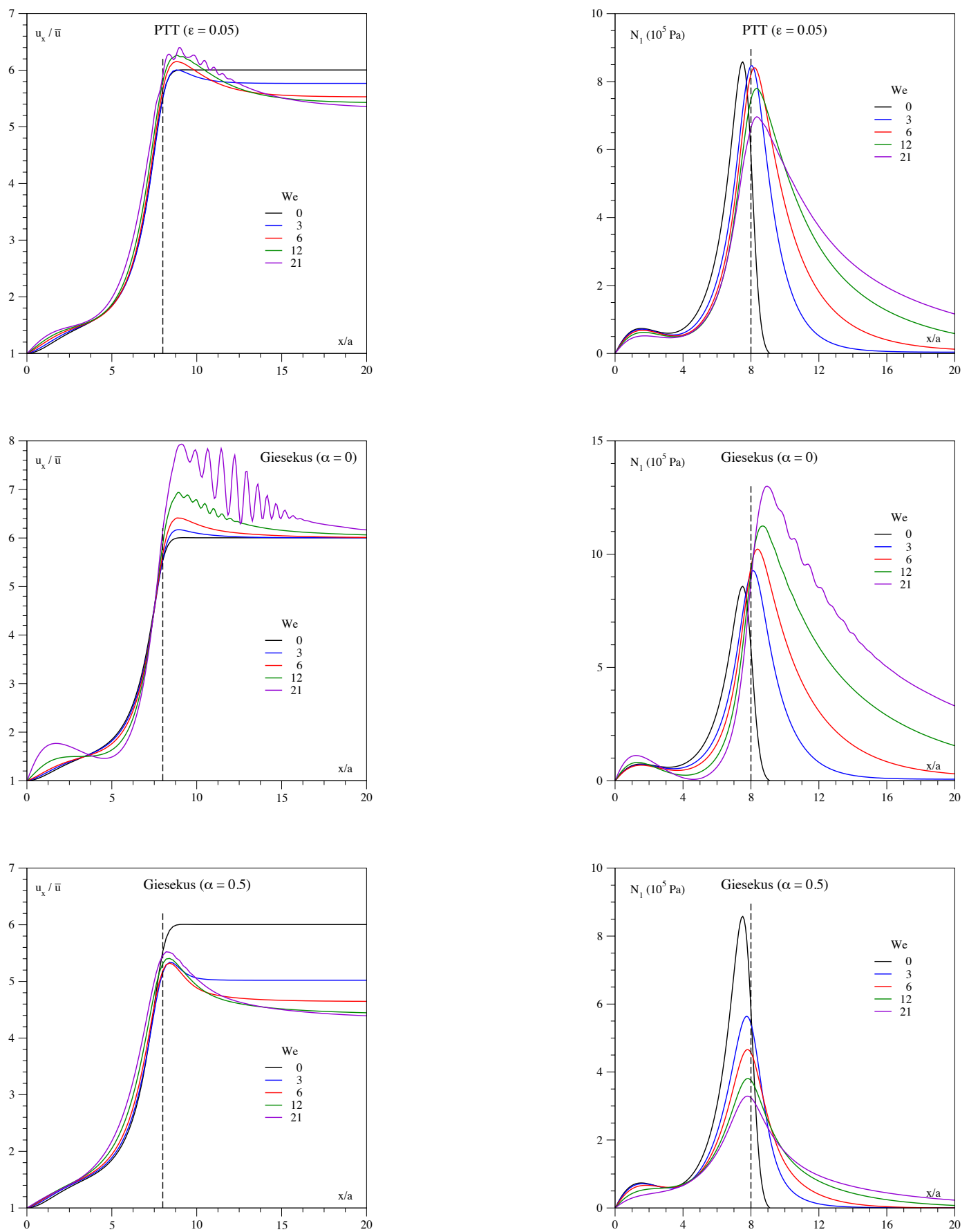

Figure 19: 4:1 contraction. $u_{x} / \bar{u}$ vs. $x / a$ along the axis of symmetry. Phan-Thien-Tanner, $\epsilon=0.05$ and Giesekus, $\alpha=0,0.5$ liquids.

Figure 20: 4:1 contraction. $N_{1}$ vs. $x / a$ along the axis of symmetry. Phan-Thien-Tanner, $\epsilon=0.05$ and Giesekus, $\alpha=0,0.5$ liquids. 


\section{References}

[1] Baaijens, F.P.T. Mixed finite element methods for viscoelastic flow analysis: a review. Journal of Non-Newtonian Fluid Mechanics 1998;79:361

[2] Baaijens F.P.T., Hulsen, M.A., Anderson, P.D. The Use of Mixed Finite Element Methods for Viscoelastic Fluid Flow Analysis. In: Stein E., de Borst R., Hughes T.J.R., editor. Encyclopedia of Computational Mechanics. John Wiley \& Sons, Ltd, Chichester 2004;3 (Fluids);Ch. 14:481

[3] Becker R., Capatina D., Joie J. A dG method for the Stokes equations related to nonconforming approximations, Research report, INRIA Concha/UPPA 2009.

URL http://hal .inria.fr/inria-00380772/en/

[4] Becker R., Capatina D. Finite element discretization of the Giesekus model for polymer flows, in: Enumath, Uppsala (Sweden), 2009.

[5] Becker R., Capatina D., Graebling D., Joie J. Nonconforming finite element approximation of the Giesekus model for polymer flows, Computers \& Fluids 2011;46:142.

[6] Coronado O.M., Arora D., Behr M., Pasquali M. A simple method for simulating general viscoelastic fluid flows with an alternate log-conformation formulation. Journal of NonNewtonian Fluid Mechanics 2007;147:189.

[7] Crouzeix M., Raviart, P.A. Conforming and nonconforming finite element methods for solving the stationary Stokes equations. I. Rev. Française Automat. Informat. Recherche Opérationnelle Sér. Rouge 1973;7 (R-3):33.

[8] Damanik H., Hron J., Ouazzi A., Turek S. A monolithic FEM approach for the log-conformation reformulation (lcr) of viscoelastic flow problems. Journal of Non-Newtonian Fluid Mechanics 2010;165:1105.

[9] D’Avino G., Maffettone P.L., Hulsen M.A., Peters G.W.M. Numerical simulation of planar elongational flow of concentrated rigid particle suspensions in a viscoelastic fluid. Journal of NonNewtonian Fluid Mechanics 2008;150:65

[10] Dou H.-S., Phan-Thien N. The flow of an Oldroyd-B fluid past a cylinder in a channel: adaptive viscosity vorticity (DAVSS$\omega)$ formulation. Journal of Non-Newtonian Fluid Mechanics 199;87:47.

[11] Étienne J., Hinch E.J., Li J. A Lagrangian-Eulerian approach for the numerical simulation of free-surface flow of a viscoelastic material. Journal of Non-Newtonian Fluid Mechanics 2006;136:157.

[12] Fattal R., Kupferman R. Constitutive laws for the matrixlogarithm of the conformation tensor. Journal of NonNewtonian Fluid Mechanics 2004;123:281.

[13] Fortin M., Fortin A. A new approach for the FEM simulation of viscoelastic flows. Journal of Non-Newtonian Fluid Mechanics 1989;32:295.

[14] Giesekus H. A simple constitutive equation for polymer fluids based on the concept of deformation-dependent tensorial mobility. Journal of Non-Newtonian Fluid Mechanics 1982;11:69.

[15] Giesekus H. Consitutive equations for polymer fluids based on the concept of configuration-dependent molecular mobility: a generalized mean-configuration model. Journal of NonNewtonian Fluid Mechanics 1985;17:349.

[16] Guénette R., Fortin M. A new mixed finite element method for computing viscoelastic flows. Journal of Non-Newtonian Fluid Mechanics 1995;60:27.

[17] Grmela M., Carreau P.J. Conformation tensor rheological models. Journal of Non-Newtonian Fluid Mechanics 1987;23:271.

[18] Hulsen M.A., Fattal R., Kupferman R. Flow of viscoelastic fluids past a cylinder at high Weissenberg number: Stabilized simulations using matrix logarithms. Journal of Non-Newtonian Fluid Mechanics 2005;127:27.

[19] Keunings R. On the high Weissenberg number problem. Journal of Non-Newtonian Fluid Mechanics 1986;20:206.

[20] Khan S.A., Larson, R.G. Comparison of simple constitutive equations for polymer melts in shear and biaxial and unixial extensions. Journal of Rheology 1987;31:207.

[21] King R.C., Apelian M.R., Armstrong R.C., Brown R.A. Numerically stable finite element techniques for viscoelastic calculations in smooh and singular geometries. Journal of NonNewtonian Fluid Mechanics 1988;29:147.

[22] Kwon Y. Finite element analysis of planar 4:1 contraction flow with the tensor-logarithmic formulation of differential constitutive equations. Korea-Australia Rheology Journal 2004;16:183.

[23] Lee Y.-J., Xu J. New formulations, positivity preserving discretizations and stability analysis for non-Newtonian flow models. Computers methods in applied mechanics and engineering 2006;195:1180.

[24] Lesaint P., Raviart P.A. On a finite element method for solving the neutron transport equation. In: de Boor C.A., editor. Mathematical Aspects of Finite Element Methods in Partial Differential Equations. Academic Press 1974.

[25] Lim F.J., Schowalter W.R. Pseudo-spectral analysis of the stability of pressure-driven flow of a Giesekus fluid between parallel planes. Journal of Non-Newtonian Fluid Mechanics 1987;26:135

[26] Marchal J.M., Crochet M.J. A new mixed finite element for calculating viscoelastic flow. Journal of Non-Newtonian Fluid Mechanics 1987;26:77.

[27] Oliveira P.J., Pinho F.T. Analytical solution for fully developed channel and pipe flow of Phan-Thien-Tanner fluids. Journal of Fluid Mechanics 1999;387:271.

[28] Owens R.G., Phillips T.N. Computational rheology. London: Imperial College Press 2002.

[29] Owens R.G., Chauvière C., Philips T.N. A locally-upwinded spectral technique (LUST) for viscoelastic flows. Journal of Non-Newtonian Fluid Mechanics 2002;108:49.

[30] Phan-Thien N., Tanner R.I. A new constitutive equation derived from network theory. Journal of Non-Newtonian Fluid Mechanics $1977 ; 2: 353$

[31] Phan-Thien, N. A nonlinear network viscoelastic model. Journal of Rheology 1978;22:259.

[32] Quinzani L.M., Armstrong R.C., Brown R.A. Birefringence and laser-Doppler velocimetry (LDV) studies of viscoelastic flow through a planar contraction. Journal of Non-Newtonian Fluid Mechanics 1994;52:1.

[33] Rajagopalan D., Armstrong, R.C., Brown R.A. Finite element methods for calculation of steady, viscoelastic flow using constitutive equations with a Newtonian viscosity. Journal of NonNewtonian Fluid Mechanics 1990;36:159.

[34] Rannacher R., Turek S. Simple nonconforming quadrilateral Stokes element. Numerical Methods Partial Differential Equations 1992;8(2):97.

[35] Sun J., Phan-Thien N., Tanner R.I. An adaptive viscoelastic stress splitting scheme and its applications: AVSS/SI and AVSS/SUPG. Journal of Non-Newtonian Fluid Mechanics 1996;65:75.

[36] Walters K., Webster M.F. The distinctive CFD challenges of computational rheology. International Journal for Numerical Methods of Fluids 2003;43:577.

[37] van der Zanden J., Hulsen M. Mathematical and physical requirements for successful computations with viscoelastic fluid models. Journal of Non-Newtonian Fluid Mechanics 1988;29:93.

[38] Xue S.-C., Phan-Thien N., Tanner R.I. Three dimensional 
numerical simulations of viscoelastic flows through planar contractions. Journal of Non-Newtonian Fluid Mechanics 1998;74:195. 